Few-Body Systems 15, 1-13 (1993)

\title{
Three-Hadron Form Factor via Quark-Triangle Loop
}

\author{
W-Y. P. Hwang ${ }^{1}$ and A. N. Mitra ${ }^{2}$ \\ ${ }^{1}$ Department of Physics, National Taiwan University, Taipei, Taiwan 10764, Republic of China \\ ${ }^{2}$ Department of Physics and Astrophysics, University of Delhi, Delhi 110007, India
}

\begin{abstract}
An explicit construction in a manifestly Lorentz-invariant and cyclically symmetric form is given for a three-hadron $(H)$ coupling through their respective $(q \bar{q})$ constituents interacting via a quark triangle loop under the most general conditions of unequal mass kinematics at both the elementary quark and composite-hadron levels. The only ansatz employed is that the $H q \bar{q}$ vertex function $\Gamma_{H}$ at each vertex $i$ is a function of $\hat{q}_{i}$, the relative momentum transverse to the corresponding hadron momentum $P_{i}$, an assumption which can be justified from several independent angles. The resulting structure $\Gamma_{I I} \approx D(\hat{q}) \otimes$ $\phi(\hat{q})$, with $D(\hat{q})$ being a model-independent function, has proved crucial for an explicit integration over the time-like component of the loop momentum, and thus provided an analytical, cyclically symmetric structure free of overlapping pole singularities. The applicational potential of this quantity is outlined.
\end{abstract}

\section{Statement of the Problem}

The internal quark structures of $q \bar{q}$ hadrons $(H)$ are compactly expressed by their $H q \bar{q}$ vertex function $\Gamma_{H}$ representing the principal dynamical vehicle through which these hadrons interact with their own species [1]. In the lowest order these interactions take place through the simplest quark loops of which the 3-quark type (triangular) is particularly appropriate for a large class of hadronic processes such as $H \rightarrow H^{\prime}+\gamma, H \rightarrow H^{\prime}+H^{\prime \prime}$, etc. The calculation of such processes through quark loops corresponds roughly to their "tree-diagrams" in which the effects of higherorder interactions (e.g., those corresponding to criss-cross gluon lines inside the quark triangle) are neglected in the first instance. More importantly these basic hadronic processes, which are often observable by themselves, may, through suitable off-shell extensions, be also incorporated as building blocks of bigger hadronic processes such as hadron-hadron scattering via hadron exchanges (Reggeized or otherwise), $p \bar{p}$ annihilation into hadrons [1], semi-leptonic and non-leptonic decays of heavy mesons (involving more than one loop), the Sullivan process for deepinelastic scattering $[2,3]$, and so on. What makes a 3-hadron vertex through a quark-triangle loop a particularly attractive object of study is its compact mathematical structure with cyclic symmetry under the most general conditions of 


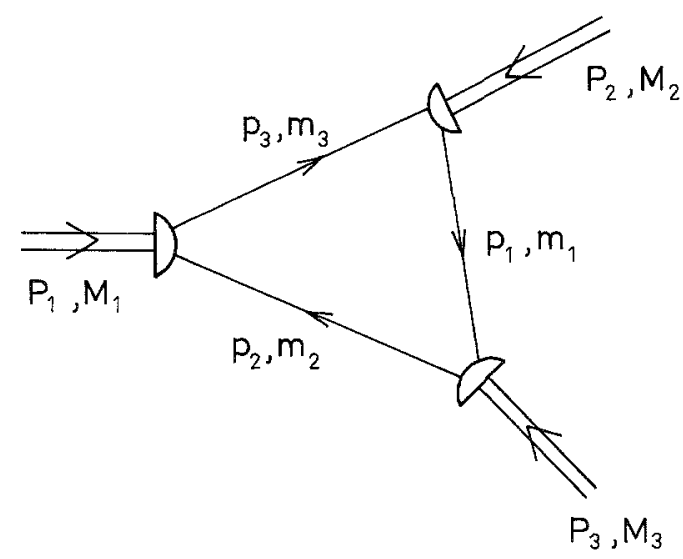

Fig. 1. Three-hadron form factor via quark triangle loop. The hadron 4-momenta are all drawn inwards $\left(P_{1}+P_{2}+P_{3}=0\right)$ and the quark lines are in cyclic order

unequal-mass kinematics (at both the hadron and quark levels) which in turn greatly expands its applicational potential to a large number and variety of cases of practical interest.

Since the key ingredient of the 3-hadron form factor is the hadron-quark vertex function $\Gamma_{H}$, as illustrated by Fig. 1 , a concrete calculational handle requires a sufficiently realistic, yet general enough ansatz of its structure to facilitate the loop integration. In this regard, a point-like structure $\left(\Gamma_{H}=\right.$ constant $)$ would be too simplistic to be physically significant, while a fully extended four-dimensional (4D) structure $\Gamma_{H}(q, P)$, (where $P_{\mu}$ is the total hadron 4-momentum and $q_{\mu}$ the relative 4-momentum of the quarks inside the hadron), would be too general to have much practical value for concrete calculations. An intermediate situation is represented by a structure in which the relative momentum $q_{\mu}$ appears in a form transverse to the total momentum $P_{\mu}$, viz. as $\Gamma_{H}(\hat{q})$, where

$$
\hat{q}_{\mu}=q_{\mu}-\frac{q \cdot P}{P^{2}} P_{\mu} .
$$

Such an ansatz can be motivated from several different angles, all of which emphasize the basically $3 \mathrm{D}$ nature of the $q \bar{q}$ interaction within the (composite) hadron structure:

(i) It gives a physical meaning to the interaction of the quark constituents in their respective mass shells (even in the intermediate states), a result which can also be justified within the tenets of local field theory [4].

(ii) It arises from the concept of an instantaneous interaction [5] between the quark constituents in accordance with the Markov-Yukawa picture of transversality to the composite 4-momentum $P_{\mu}[6]$.

(iii) It is the only structure consistent with the compatibility of the Bethe-Salpeterequation (BSE) kernel $K$ for a pair of Dirac equations for two particles under mutual interaction [7].

(iv) Finally it is a direct consequence of the ansatz $[8,9]$ of a 3D support $(\hat{K})$ to the BSE kernel $K$ for a $q \bar{q}$ system in a fully Lorentz-covariant manner, which in turn gives rise to an exact interconnection [8] between two alternative forms of the BSE: a $3 D$ reduction of the form $D \phi=\int \hat{K} \phi$ where all quantities are functions of $\hat{q}$, and a reconstructed 4D form that now supplies the definition of 
$\Gamma_{H}(\hat{q})$ in the form $D \otimes \phi .(D(\hat{q})$ and $\phi(\hat{q})$ are thus the $3 \mathrm{D}$ denominator function and wave function, respectively.) There is one more point that provides an observational support to the above ansatz: Namely, the $3 \mathrm{D}$ form $D \phi=\int \hat{K} \phi$ of the BSE is in conformity with the observed $O(3)$-like spectra [10] of hadrons, while a more general form with a 4D support for the kernel [11] would predict $O(4)$-like spectra, in disharmony with observation [10]. Though this fact has been known since the seventies [12], it is perhaps not out of place to stress it again, in the context of renewed attempts [13] to fit spectroscopic data with $O(4)$-like gluon propagator inputs for the coupled SDE and BSE.

Fortified by these considerations, we shall henceforth employ the ansatz hereafter termed the covariant-instantaneity ansatz (CIA) $[8,9]$ which gives the structure at each of the three vertices of Fig. 1, as a basis for the evaluation of the $H H H$ vertex, under completely general conditions of unequal-mass kinematics, which is the principal object of this paper. Specifically we shall show how the structure $\Gamma_{H} \sim D \phi$ facilitates a compact and transparent Lorentz-invariant representation of the $\mathrm{HHH}$ form factor in a sufficiently general manner as well in conformity with cyclic symmetry. The principal result is that the integration over the time-like component of the internal loop momentum $p_{i}$ can be carried out exactly in a form that brings out the cancellation of the colliding pole effects (Landau-Cutkowsky singularities) as a direct consequence of the $D \otimes \phi$ structure of $\Gamma_{H}$, thus preventing the free propagation of quarks in intermediate states [8]. On the other hand, the non-locality of the $H q \bar{q}$ vertex function $\Gamma(\hat{q})$ causes in general an additional complexity problem for the 3-hadron form factor over and above those dictated by the standard dispersion relations when the physical thresholds for real particle production are crossed. This problem was discussed recently in the context of a special example (the $\rho \pi \pi$ coupling constant), and was traced to the effect of the confining nature of the $q \bar{q}$ interactions. However, pending a proper understanding of this non-locality effect, a simple prescription after Goldberger and Treiman [15], namely a symmetrized average of $\mid$ in $\rangle$ and $\mid$ out $\rangle$ intermediate states, "in order to maintain the proper reality conditions at all stages of the approximation" [15], was adopted [14] and a real value obtained for $g_{\rho \pi \pi}$. We shall follow the same prescription here, in order to circumvent any complexity problem due to the non-local structure of the quantities $\Gamma_{H}(\hat{q})$.

We end this section by defining the full structure of the 3-hadron amplitude corresponding to Fig. $1[1,16]$, with all momenta incoming $\left(P_{1}+P_{2}+P_{3}=0\right)$, viz.,

$$
\begin{aligned}
A(3 H)= & \frac{2 i}{\sqrt{3}}(2 \pi)^{8} \int d^{4} p_{i} \Gamma_{1}\left(\hat{q}_{1}\right) \Gamma_{2}\left(\hat{q}_{2}\right) \Gamma_{3}\left(\hat{q}_{3}\right) \\
& \times \operatorname{Tr}\left\{O_{1} S_{F}\left(p_{3}\right) O_{2} S_{F}\left(p_{1}\right) O_{3} S_{F}\left(p_{2}\right)\right\},
\end{aligned}
$$

exhibiting the cyclic symmetry explicitly. Here, for simplicity, we have factored out a Dirac matrix $O_{i}$ sitting at each $H q \bar{q}$ vertex (which also includes a possible flavour matrix), so that the reduced vertex function $\Gamma_{i}$ has the form $[8,14]$

$$
\Gamma_{i}\left(\hat{q}_{i}\right)=-i N_{i} D_{i}\left(\hat{q}_{i}\right) \otimes \phi_{i}\left(\hat{q}_{i}\right)(2 \pi)^{-5 / 2} .
$$

Here, $N_{i}$ is the BS normalizer for the hadron $i[12,8,14]$, and the fuller definitions of $D_{i}$ and $\phi_{i}$ are given in Sect. 3. The colour factor and the effect of reversing the 
loop direction are represented by the factor $2 / \sqrt{3}$ in front, and $(2 \pi)^{8}$ is the net result of an additional $(2 \pi)^{4}$ factor at each vertex (due to BS normalization $[12,16]$ ) and a routine division by an overall factor $(2 \pi)^{4} \delta^{4}\left(P_{1}+P_{2}+P_{3}\right)$ appropriate for a reduced amplitude. For subsequent purposes it is more convenient to express the amplitude (1.1) in the alternative form,

$$
\begin{aligned}
A(3 H) & =-\frac{2 i}{\sqrt{3}} \sqrt{2 \pi} N_{1} N_{2} N_{3} \int d^{4} p_{i} \prod_{1}^{3}\left(D_{i} \otimes \phi_{i} / \Delta_{i}\right)[T R], \\
{[T R] } & =\operatorname{Tr}\left\{O_{1}\left(m_{3}-i \gamma \cdot p_{3}\right) O_{2}\left(m_{1}-i \gamma \cdot p_{1}\right) O_{3}\left(m_{2}-i \gamma \cdot p_{2}\right)\right\}, \\
\Delta_{i} & =p_{i}^{2}+m_{i}^{2} .
\end{aligned}
$$

In Sect. 2 we define our notations, phase conventions and collect several kinematical relations among the quantities involved. Sect. 3 gives a complete derivation of the 3-hadron amplitude by carrying out the integration over the time-like component of the loop momentum in terms of null-plane variables, and express the result as a simple quadrature free of internal singularities. Sect. 4 discusses briefly the model-independent aspect of the derivation and its rich applicational potential. On the other hand, in keeping with the generality of this derivation, specific applications are relegated to future publications.

\section{Kinematical Considerations}

The momentum and phase conventions for the external (hadron) and internal (quark) lines are specified in Fig. 1, viz., the 4-momenta $P_{i \mu}=P_{i}$ of the hadrons (masses $M_{i}$ ) are all drawn inwards and the 4-momenta $p_{i \mu}=p_{i}$ of the quarks (masses $m_{i}$ ) are drawn cyclically. Let the relative momentum of the quarks inside hadron $i$ be $q_{i}=q_{i \mu}$, and let $\hat{q}_{i}=\hat{q}_{i \mu}$ be its component transverse to $P_{i \mu}$. Thus with $(i j k)=(123)$ (cyclic) we have the relations

$$
\begin{gathered}
-P_{k}=P_{i}+P_{j}=P_{i j}, \quad P_{k}^{2}=-M_{k}^{2}, \\
P_{k}=-P_{i j}=p_{j}-p_{i}, \\
q_{k}=\hat{\mu}_{i j} p_{j}+\hat{\mu}_{j i} p_{i}, \\
\hat{q}_{k \mu}=q_{k \mu}-\frac{q_{k} \cdot P_{k}}{P_{k}^{2}} P_{k \mu} .
\end{gathered}
$$

The quantities $\hat{\mu}_{i j}$, which may be regarded as the fractional momenta carried by the constituent quarks within a specified hadron $H_{k}\left(P_{k}\right)$, are given a Lorentz-invariant meaning according to the Wightman-Garding definitions [17],

$$
\hat{\mu}_{i j}=\frac{1}{2}\left\{1+\frac{m_{i}^{2}-m_{j}^{2}}{M_{k}^{2}}\right\} .
$$

Analogously, corresponding to a break-up $H_{k} \rightarrow H_{i}+H_{j}$ (real or virtual), we can define the hadronic counterparts $\left(Q_{k \mu}, \hat{m}_{i j}\right)$ of $\left(q_{k \mu}, \hat{\mu}_{i j}\right)$ as

$$
\begin{gathered}
Q_{k \mu}=\hat{m}_{i j} P_{j}-\hat{m}_{j i} P_{i}, \quad-P_{k}=P_{i}+P_{j}, \\
\hat{m}_{i j}=\frac{1}{2}\left\{1+\frac{M_{i}^{2}-M_{j}^{2}}{M_{k}^{2}}\right\} .
\end{gathered}
$$


Note that the difference of sign in $P_{i}$ of Eq. (2.6) viv-à-vis $p_{i}$ of Eq. (2.3) arises from our respective phase conventions for them. Similarly

$$
\hat{Q}_{\kappa \mu}=Q_{\kappa \mu}-\frac{Q_{k} \cdot P_{k}}{P_{k}^{2}} P_{k \mu}, \quad \hat{Q}_{k} \cdot P_{k}=0 .
$$

Note that if all the hadrons $H_{i}$ are on their respective mass shells then $Q_{k} \cdot P_{k}$ vanishes identically by virtue of the definition (2.7) of the fractional momenta, so that $\hat{Q}_{k} \equiv Q_{k}$. However, for off-shell applications of the 3-hadron vertex function $Q_{k} \cdot P_{k}$ does not in general vanish, so that the definition (2.8) for $\hat{Q}_{k}$ is the appropriate one. Next, it is necessary to express all the relative momenta $q_{i}$ in terms of a common basis momentum such as $p_{i}$ corresponding to a situation (see Sect. 3) when the quark-line $p_{i}$ is on its mass shell. Thus in a fixed $p_{i}$ basis we have

$$
\begin{gathered}
q_{k}=p_{i}+\hat{\mu}_{i j} P_{k}, \quad q_{j}=p_{i}-\hat{\mu}_{i k} P_{j}, \\
q_{i}=p_{i}-\hat{\mu}_{j k} P_{j}+\hat{\mu}_{k j} P_{k}
\end{gathered}
$$

with $i=1,2,3$, by turn in an obvious cyclic notation.

As we shall be using null-plane coordinates for integration over the loop momenta, the following representation for $\hat{q}_{i}^{2}$ is useful [8]:

$$
\hat{q}_{i}^{2}=q_{i \perp}^{2}+\frac{1}{4 M_{i \perp}^{2}}\left\{q_{i+} P_{i-}-q_{i-} P_{i+}\right\}^{2}, \quad M_{i \perp}^{2}=M_{i}^{2}+P_{i \perp}^{2} .
$$

Further, as we shall consider only coplanar processes (what somewhat restricts the applicability of the $\mathrm{HHH}$ form factor when it is considered as part of a bigger process), we can set all $P_{i \perp}=0$, so that

$$
p_{i \perp}^{2}=q_{i \perp}^{2}=p_{\perp}^{2}
$$

independent of $i$.

This simplification will turn out to be crucial for the calculation of the form factor (see Sect. 3). Finally the quantities $\hat{q}_{i}^{2}$ can be expressed in a common basis, say $p_{2}$, in accordance with (2.9)-(2.12) as

$$
\begin{aligned}
& \hat{q}_{i}^{2}=p_{\perp}^{2}+\frac{1}{4 M_{i}^{2}}\left(p_{2+} P_{i-}-p_{2-} P_{i+}\right)^{2}, \quad i \neq 2, \\
& \hat{q}_{2}^{2}=p_{\perp}^{2}+\frac{1}{4 M_{2}^{2}}\left(p_{2+} P_{2-}-p_{2-} P_{2+}+Q_{2+} P_{2-}-Q_{2-} P_{2+}\right),
\end{aligned}
$$

where we have employed the relation $(i j k=123 \mathrm{cyclic})$

$$
q_{i \mu}=p_{i \mu}+Q_{i \mu}+\left(\hat{\mu}_{j k} \hat{m}_{j k}-\hat{\mu}_{k j} \hat{m}_{k j}\right) P_{i \mu} .
$$

Several additional relations follow if the external particles are on their mass shells $\left(Q_{i} \cdot P_{i}=0\right)$

$$
P_{i \pm}= \pm \frac{M_{i}}{Q_{i}} Q_{i \pm}
$$

where

$$
M_{i}^{2}=P_{i+} P_{i-}, \quad Q_{i}^{2}=-Q_{i+} Q_{i_{-}}>0
$$

Further, we have 


$$
\begin{aligned}
Q_{i}^{2} & =\hat{m}_{j k}^{2} M_{i}^{2}-M_{j}^{2}=\hat{m}_{k j}^{2} M_{i}^{2}-M_{k}^{2} \\
& =\lambda\left(M_{1}^{2}, M_{2}^{2}, M_{3}^{2}\right) /\left(4 M_{i}^{2}\right),
\end{aligned}
$$

where $\lambda$ is the $S_{3}$-invariant triangle function of its arguments. The last step gives the important symmetry relation

$$
Q_{1} M_{1}=Q_{2} M_{2}=Q_{3} M_{3}=\frac{1}{2} \sqrt{\lambda} \cdot \text { (inv) }
$$

Again, the appearance of $P_{i \pm}$ in Eqs. (2.13) and (2.14) suggests that for subsequent purposes we shall need the structure of the $3 \times 3$ matrix $\left\{n_{i j}\right\}$, where

$$
n_{i j} \equiv P_{i+} / P_{j+} \text {. }
$$

These quantities are not all independent but are subject to the conditions

$$
n_{i j} n_{j i}=1, \quad n_{12} n_{23} n_{31}=1, \quad \sum_{i} n_{i 1}=0 .
$$

After a short algebra the matrix structure works out as

$$
\left\{n_{i j}\right\}=\left(\begin{array}{ccc}
1 & -\hat{m}_{13} \pm\left(Q_{2} / M_{2}\right) & -\hat{m}_{12} \mp\left(Q_{3} / M_{3}\right) \\
-\hat{m}_{23} \mp\left(Q_{1} / M_{1}\right) & 1 & -\hat{m}_{21} \pm\left(Q_{3} / M_{3}\right) \\
-\hat{m}_{23} \pm\left(Q_{1} / M_{1}\right) & -\hat{m}_{31} \mp\left(Q_{2} / M_{2}\right) & 1
\end{array}\right),
$$

where a two-fold ambiguity is expressed by the statement that only the upper, or only the lower, signs must be taken. It is easy to verify that Eq. (2.23) satisfies all the conditions (2.22), after taking account of Eqs. (2.18)-(2.20).

At this stage we are in a position to express the inverse propagators $\Delta_{i}$, Eq. (1.5), in the form

$$
\Delta_{i}=\omega_{i \perp}^{2}-p_{i+} p_{i-},
$$

where, using Eq. (2.12),

$$
\omega_{i \perp}^{2}=p_{\perp}^{2}+m_{i}^{2}
$$

\section{Evaluation of the $\mathrm{HHH}$ Form Factor}

With this kinematical equipment, we are now in a position to evaluate the threehadron amplitude $A(3 H)$ defined by Eqs. (1.3)-(1.5) through a sequence of steps. First, to bring out the analytic structure of $A(3 H)$ it should be adequate to temporarily suppress the trace factor $[T R]$ in Eq. (1.3), which does not contribute to any singularities whose effect, however, can be trivially included later (see end of this section). Next we define the function $D_{i}\left(\hat{q}_{i}\right)$ of Eq. (1.2) more comprehensively as follows,

$$
\begin{aligned}
D_{i}\left(\hat{q}_{i}\right) & =\Omega_{j k}\left(\hat{q}_{i}\right) \Delta_{j k}\left(\hat{q}_{i}\right), \\
\frac{1}{2} \Delta_{j k}\left(\hat{q}_{i}\right) & =\hat{q}_{i}^{2}-\lambda\left(M_{i}^{2}, m_{j}^{2}, M_{k}^{2}\right) /\left(4 M_{i}^{2}\right), \\
2 \Omega_{j k}^{-1}\left(\hat{q}_{i}\right) & =\frac{\hat{\mu}_{j k}}{\omega_{j}\left(\hat{q}_{i}\right)}+\frac{\hat{\mu}_{k j}}{\omega_{k}\left(\hat{q}_{i}\right)},
\end{aligned}
$$

where

$$
\omega_{i}^{2}(\hat{q})=\hat{q}^{2}+m_{i}^{2},
$$

and $\hat{q}$ can be any one of $\hat{q}_{i}(i=1,2,3)$. Similarly the 3D wave function $\phi(\hat{q})$ at each 
vertex is given by $[8,14]$

$$
\phi\left(\hat{q}_{i}\right)=\exp \left(-\frac{\hat{q}_{i}^{2}}{2 \beta_{i}^{2}}\right)
$$

where $\beta_{i}^{2}$ is determined by dynamics. (At this stage we wish to stress, however, that for the derivation of our principal result, Eq. (3.21), this function does not play any active role, except for providing an overall convergence factor for the resulting quadrature). These definitions are in conformity with the null-plane representations of the quantities $\hat{q}(i)$ given in Sect. 2. Next we should carry out the integration in terms of null-plane variables $x_{i}, y_{i}$ in a given basis, in accordance with the $S_{3}$-invariant measure

$$
\begin{gathered}
d^{4} p_{i}=d^{2} p_{\perp} \cdot \frac{1}{2} d x_{i}^{2} M_{i}^{2} d y_{i}, \\
x_{i}=p_{i+} / P_{i+}, \quad y_{i}=p_{i-} / P_{i-} .
\end{gathered}
$$

The central question now is: Which index $(i)$ should be chosen so as to preserve the cyclic symmetry in a general enough fashion? In this connection we should first clarify the problem of complexity (raised in Sect. 1) that might arise from an indiscriminate application of pole-integration techniques, say with respect to $d y_{i}$, since the presence of Gaussian factors $\phi_{j}, j \neq i$, with respect to $\hat{q}_{j}^{2}$, which involves both $x_{i}$ and $y_{i}$ in any basis (i) [see Eqs. (2.13) and (2.14)], will prevent a normal closure of the semi-circle in the $y_{i}$-plane. In this sense a new complexity problem that arises is directly attributable to the (Gaussian) non-locality of the hadron-quark vertex function [14]. Pending a deeper understanding of this problem we shall use the same Goldberger-Treiman (GT) prescription [15] as already employed in ref. [14], viz., for the intermediate (quark) states the symmetrized combination $(\mid$ in $\rangle+\mid$ out $\rangle) / 2$ will ensure the necessary reality. Since the quarks appear through the propagators $\Delta_{i}^{-1}$, the meaning of this prescription can be understood as follows: For each propagator we have

$$
\frac{1}{\Delta_{i}-i \varepsilon}=\mathscr{P} \frac{1}{\Delta_{i}}+i \pi \delta\left(\Delta_{i}\right)
$$

where we have depicted the imaginary part $(-i \varepsilon)$ separately from $m_{i}^{2}$. When three such propagators are multiplied together, the real part will come from two $\mathscr{P}$-terms and one $\delta$-term [note that a factor $i$ is sitting in front of Eq. (1.1)] in three different ways; the other contribution arising from the product of three $\mathscr{P}$-terms, of course, vanishes. The imaginary part, on the other hand, would come from one $\mathscr{P}$-term and two $\delta$-terms in three different ways, as well as from the product of three $\mathscr{P}$-terms. Now the GT prescription [15] amounts to taking only the first category of terms (real) and dropping the second category (imaginary). Of the latter the $D(\hat{q})$ factor in our vertex function (1.2) ensures the automatic vanishing of the product of one $\mathscr{P}$-term and two $\delta$-terms, but the product of three $\delta$-terms would have still remained in the absence of the GT prescription; its understanding is beyond the scope of this paper. As to the admissible (real) terms, these now come only from the product of two $\mathscr{P}$-terms and one $\delta$-term in three different ways, thus providing the vital clue towards maintaining the desired cyclic symmetry in the integration procedure. Namely, since the measure $d^{4} p_{i}$ is cyclically invariant, the formula (3.6) can be employed by turn in cyclic order to integrate over $d y_{i}$, in association with the corresponding $\delta$-function available for this case. 
It is therefore enough to outline the procedure with respect to basis 2 , say, in which the necessary formulae for the $q_{i}$ 's have already been listed in Eqs. (2.12)(2.14). Using the representation (2.24)-(2.25) for $\Delta_{2}$, the integral over $y_{2}$ gives

$$
\int i \pi \delta\left(\Lambda_{2}\right) d y_{2} M_{2}^{2}=(i \pi) / x_{2}
$$

with

$$
y_{2}=\xi_{2} \equiv \omega_{2 \perp}^{2} /\left(M_{2}^{2} x_{2}\right) \text {. }
$$

The next thing is to observe some remarkable simplifications that occur at this pole value of $y_{2}$, viz., the denominator functions $D_{1}$ and $D_{3}$ sitting at the opposite ends of the $p_{2}$-line in Fig. 1 will cancel out the (otherwise overlapping) singularities due to the complementary propagators $\Delta_{3}^{-1}$ and $\Delta_{1}^{-1}$ associated with $D_{1}$ and $D_{3}$, respectively.

To prove this result, the pole value $y_{2}=\xi_{2}$ from Eq. (3.10) must first be substituted in the various quantities, and in this process use should be made of several kinematical relations of Sect. 2 to give a symmetrical appearance to the resultant structure. At the $\Delta_{2}$-pole one has the following results:

$$
\begin{gathered}
\Delta_{1 ; 2}=\xi_{2} n_{32} M_{2}^{2}+x_{2} n_{23} M_{3}^{2}-2 \hat{\mu}_{21} M_{3}^{2}, \\
\Delta_{3 ; 2}=-\xi_{2} n_{12} M_{2}^{2}-x_{2} n_{21} M_{1}^{2}-2 \hat{\mu}_{23} M_{1}^{2}, \\
\Delta_{2,2}=0, \\
i \neq 2: \quad \hat{q}_{i ; 2}^{2}=-m_{2}^{2}+\frac{1}{4 M_{i}^{2}}\left(M_{2}^{2} \xi_{2} n_{i 2}+x_{2} n_{2 i} M_{i}^{2}\right)^{2}, \\
i=2: \quad \hat{q}_{2 ; 2}^{2}=-m_{2}^{2}+M_{2}^{2} \xi_{2} x_{2}+\left(M_{2} x_{2}-M_{2} \xi_{2}+2 Q_{2}\right)^{2}, \\
D_{1}\left(\hat{q}_{1 ; 2}\right)=\frac{1}{2} \Omega_{23}^{(1 ; 2)}\left[\left(\frac{M_{2}^{2}}{M_{1}^{2}} \xi_{2} n_{12}+x_{2} n_{21}\right)^{2}-4 \hat{\mu}_{23}^{2}\right] M_{1}^{2}, \\
D_{3}\left(\hat{q}_{3 ; 2}\right)=\frac{1}{2} \Omega_{12}^{(3 ; 2)}\left[\left(\frac{M_{2}^{2}}{M_{3}^{2}} \xi_{2} n_{32}+x_{2} n_{23}\right)^{2}-4 \hat{\mu}_{21}^{2}\right] M_{3}^{2} .
\end{gathered}
$$

The index 2 after the semi-colon indicates that the quantity has been evaluated at the $\Delta_{2}$-pole and the quantity $\Omega_{i j}$ has been renamed as $\Omega_{i j}^{(k)}$ to keep track of the different cyclic possibilities. It is now clearly seen that $D_{1}$ contains $\Delta_{(3 ; 2)}$ as a factor; similarly $D_{3}$ has $\Delta_{(1 ; 2)}$ as a factor. This proves our assertion.

Before collecting these results the only other quantity remaining to be evaluated at the $\Delta_{2}$-pole is $D_{2}(\hat{q})$ which, from the topology of Fig. 1, is situated opposite (not adjacent) to the $\Delta_{2}$-pole, so that there is no question of any overlapping poles in this case. The results are

$$
\begin{aligned}
& D_{2 ; 2} \equiv D_{2}\left(\hat{q}_{2 ; 2}\right)=\frac{1}{2} \Omega_{31}^{(2 ; 2)}\left\{\left(x_{2} M_{2}-\xi_{2} M_{2}+2 Q_{2}\right)^{2}\right. \\
& \left.+4 M_{2}^{2} x_{2} \xi_{2}-\lambda\left(M_{2}^{2}, M_{3}^{2}, m_{1}^{2}\right)\right\} \text {, } \\
& \mathscr{D}_{1 ; 2} \equiv \frac{D_{1}\left(\hat{q}_{1 ; 2}\right)}{\Delta_{3 ; 2}}=\frac{1}{2} \Omega_{23}^{(1 ; 2)}\left\{-\left(\xi_{2} n_{12} M_{2}^{2}+M_{1}^{2} x_{2} n_{21}\right) 2 \hat{\mu}_{23} M_{1}^{2}\right\} \text {, } \\
& \mathscr{D}_{3 ; 2} \equiv \frac{D_{3}\left(\hat{q}_{3 ; 2}\right)}{\Delta_{1 ; 2}}=\frac{1}{2} \Omega_{12}^{(3 ; 2)}\left\{+\left(\xi_{2} n_{32} M_{2}^{2}+M_{3}^{2} x_{2} n_{23}\right) 2 \hat{\mu}_{21} M_{3}^{2}\right\} \text {. }
\end{aligned}
$$


The product of the wave functions $\phi_{1} \phi_{2} \phi_{3}$ at the $\Delta_{2}$-pole can be compactly expressed as

$$
\Phi_{2}\left(x_{2}, \xi_{2}\right)=\exp \left(-\frac{1}{2} \sum_{1}^{3} \hat{q}_{j ; 2}^{2} \beta_{j}^{-2}\right),
$$

and similarly for the product of the reduced denominator functions

$$
\mathscr{D}_{R 2}\left(x_{2}, \xi_{2}\right)=D_{2 ; 2} \mathscr{D}_{1 ; 2} \mathscr{D}_{3 ; 2} \text {. }
$$

The integration can now be expressed in terms of $\xi_{2}, x_{2}$ after taking account of Eqs. (3.6) and (3.9) as

$$
\int d^{4} p_{2} i \pi \delta\left(\Delta_{2}\right)=\int i \pi^{2} M_{2}^{2} d x_{2} d \xi_{2}
$$

The limits of the $\xi_{2}$ and $x_{2}$ integrations are given by

$$
\frac{m_{2}^{2}}{M_{2}^{2}\left|x_{2}\right|} \leq\left|\xi_{2}\right|<\infty, \quad-\infty<x_{2}<\infty .
$$

The same procedure can be repeated in a cyclically symmetric way for the other two terms proportional to $i \pi \delta\left(\Delta_{3}\right)$ and $i \pi \delta\left(\Delta_{1}\right)$, respectively. Collecting all the results we have

$$
A(3 H)=2 \sqrt{\frac{2 \pi}{3}} N_{1} N_{2} N_{3} \sum_{1}^{3} \iint M_{i}^{2} \pi^{2} d x_{i} d \xi_{i}[T R]_{i} \mathscr{D}_{R i} \otimes \phi_{i} .
$$

Here we have formally represented the effect of the trace factor [TR] by indexing it with $i$, implying that it must also be evaluated at the $\Delta_{i}$-pole. However, an equally compact cyclically symmetric structure in terms of concrete functions like $\mathscr{D}_{i}$ and $\phi_{i}$ is not so practicable, since most cases of physical interest are those with different types of $O_{i}$ at the different vertices. Thus the form (3.21) provides about as much formal cyclic symmetry in the presence of $O_{i}$-matrices as can be expected without specifying their actual content. Perhaps the only two possible exceptions where a concrete structure can be written down in an equally compact form are the cases of all three scalars $\left(O_{i}=i \gamma \cdot \hat{q}_{i}\right)$ or all three vectors $\left(O_{i}=i \gamma \cdot \hat{\varepsilon}_{i}\right)$. On the other hand, the trace-factor is innocuous insofar as it does not modify the analytic structure of the $A(3 H)$ amplitude, which continues to be governed by the $\mathscr{D}_{i}$ and $\phi_{i}$ functions in Eq. (3.21).

\section{Discussion of General Applicability}

Our central result for the 3-hadron form factor is Eq. (3.21) in which the various quantities in the integrand are defined analytically in terms of polynomials and Gaussians in the $\left(x_{i}, \xi_{i}\right)$ variables, so that the entire structure is free from internal singularities and fully determinate in terms of external invariants $\left(M_{i}, Q_{i}\right)$. The only condition under which it is valid is that the external (hadron) lines are coplanar, which represented the only constraint on the incorporation of this element into a bigger structure involving other hadrons. Before looking for possible applications of this quantity the first question that should be asked is: How much of this derivation is model-independent? The answer lies in the structure $\Gamma_{H} \sim D \otimes \phi$ of 
the $H q \bar{q}$ vertex function which is a direct consequence of the $3 \mathrm{D}$ support to the BS kernel $[8,9]$, expressed through the transverse momentum $\hat{q}_{\mu}$ at each vertex. Though we have related this structure to the above BS dynamics, this aspect of the latter is general enough to receive support from other angles as well. Indeed, we have listed several other independent lines of the approach in Sect. 1 [4-7], all of which seem to converge towards a very similar $q$-dependence for $\Gamma_{H}$ (when Lorentz-covariance is taken into account), though to the best of our knowledge the specific form $\Gamma_{H} \sim D \otimes \phi$ has not been exploited in these approaches. In any case, the spirit of the CIA $[8,9]$, which readily offers this factorized form, has enough in common with these alternative approaches $[4,7]$ to warrant the conclusion that the $D \otimes \phi$ structure for $\Gamma_{H}$ is a fairly model-independent one. What we have exploited in the derivation of $A(3 H)$ is the structure of the $D$-function which is quite universal, indeed kinematic, and plays the role of a denominator function in a relativistic (3D) Schrödinger equation. Indeed, it is this quantity that has played the key role in an exact integration over the time-like component of the loop-momentum and has been entirely responsible for eliminating the overlapping pole effects, so as to give rise to a resultant polynomial function in $\left(x_{i}, \xi_{i}\right)$ variables. In this derivation the $\phi$-function (which is of course model-dependent, and may be regarded as the $3 \mathrm{D}$ wave function of the corresponding Schrödinger equation), has played no active role, except for providing an overall convergence factor for the subsequent $\left(x_{i}, \xi_{i}\right)$ integrations. It is this feature of the derivation of $A(3 H)$ under completely general conditions of unequal mass kinematics at both the quark and hadron levels, that lends it a sufficient degree of generality for applicational purposes, without a priori commitment to a particular type of $q \bar{q}$ wave function $\phi$, as long as it is a Lorentz-covariant $3 \mathrm{D}$ quantity. The BS normalizers $N_{i}$ can be calculated in a standard fashion [18], and are also listed in the recent literature $[8,14,19]$.

In the spirit of this generality, this paper by itself was not intended for any specific application per se, but some possibilities can be readily listed. The simplest class is of that of (strong-interaction) decay of a resonance $\left(H_{1}\right)$ into two lighter hadrons $\left(\mathrm{H}_{2}, \mathrm{H}_{3}\right)$ under kinematically allowed conditions (this signature is also carried by the external variables $Q_{i}, M_{i}$ inside the integral (3.21)). The amplitude $A(3 H)$ can also be adapted to include semi-leptonic or electromagnetic processes, generically expressed by $H_{1} \rightarrow H_{2}+\gamma$ (where $\gamma$ is real or virtual), after replacing the vertex function $\Gamma_{3}$ by unity and taking the corresponding $O_{i}$ as $i \gamma_{\mu}$ (for $V$ ) or as $i \gamma_{\mu} \gamma_{5}$ (for $A$ ) as the case may be. The signature of virtuality is carried by $M_{3}^{2}=t$. Another class of processes which are of considerable interest because of a rich interplay of unequalmass kinematics are the weak decays of $Q \bar{q}$ mesons into a pair of $q \bar{q}$ types, or even of $Q^{\prime} \bar{q}+q \bar{q}$, if $Q$ is more massive than $Q^{\prime}$ (e.g., in a $b \rightarrow c$ transition). Some possibilities are listed in Fig. 2 for the decay of a $Q \bar{q}$ meson into a pair of $q \bar{q}$ types via $W$-exchange and/or annihilation. Despite the presence of two loops, the huge mass of $W$ causes a trivial factoring out of the two-quark loop in favour of the corresponding weak amplitude, say $f_{H_{1}}$, and the triangle structure for the rest appears with $H_{1}$ playing the role of $W$, as indicated above. Identical considerations apply to spectator diagrams for non-leptonic decays as well (these are not illustrated). As an example of the potential importance of unequal-mass kinematics at the quark level, we may cite the experimental discrepancy [20] of the vector form factors in the semi-leptonic process $D \rightarrow K^{*} e v$ with theoretical models [21] that 


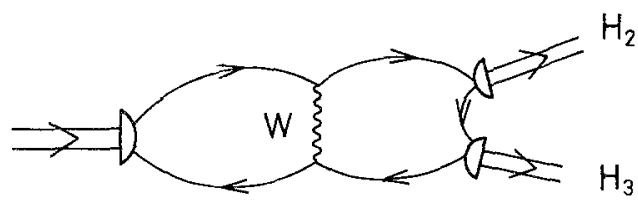

$H_{1} \equiv Q q$

(a)

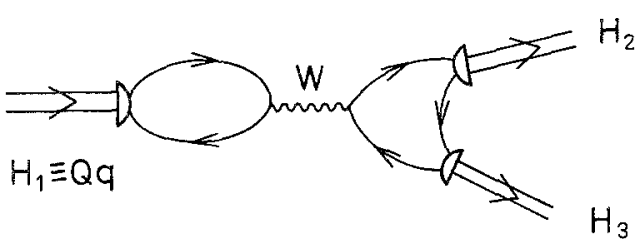

(b)

$\mathrm{H}_{1} \rightarrow \mathrm{H}_{2} \mathrm{H}_{3}$

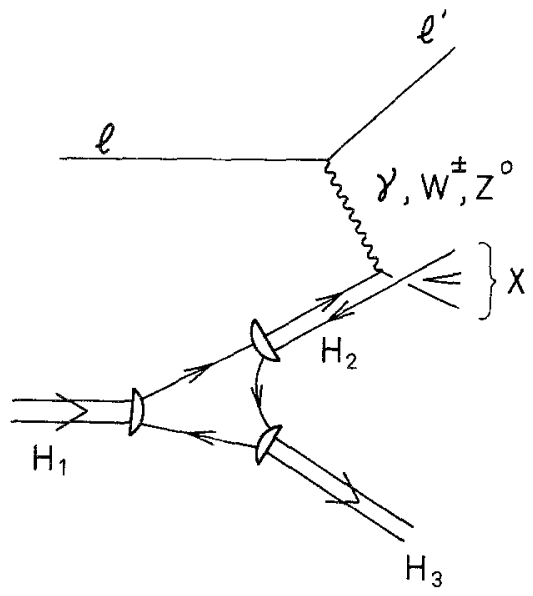

Fig. 2. Two-body decay of a heavy $Q \bar{q}$ hadron $\left(H_{1}\right)$ into a pair of lighter ones $\left(H_{2}, H_{3}\right)$ via $W$ boson exchange (a) and annihilation (b). For explanation, see Sect. 4

Fig. 3. Sullivan process, which may contribute to deepinelastic scattering $l+H_{1} \rightarrow l^{\prime}+X$. The three-hadron form factor with $\mathrm{H}_{2}$ off-shell is needed as an input

prefer to represent the intermediate states through suitable meson propagators. On the other hand, a crucial role of the appropriate quark triangle, with a considerable effect of the unequal masses of the constituents involved, seemed to be strongly indicated in resolving the discrepancy [22].

We would also like to draw attention to the so-called Sullivan process [2] in which the $3 H$ form factor can play a crucial role, by folding the latter into the electromagnetic break-up of the pion into its quark constituents. An example of the Sullivan process is illustrated in Fig. 3, in which the hadron $\mathrm{H}_{2}$ emitted by $\mathrm{H}_{1}$ is struck (and smashed into debris) by the probing particle. We might mention in this connection that the original Sullivan process has been considerably generalized $[3,23]$ in recent years to include other intermediate hadrons as an effective alternative to the inclusion of higher Fock states, while staying in the valence-quark domain for low and moderate $Q^{2}$. In this way, it has been found $[3,23]$ that valence- and sea-quark distributions for a hadron can be reproduced reasonably well. Such a 


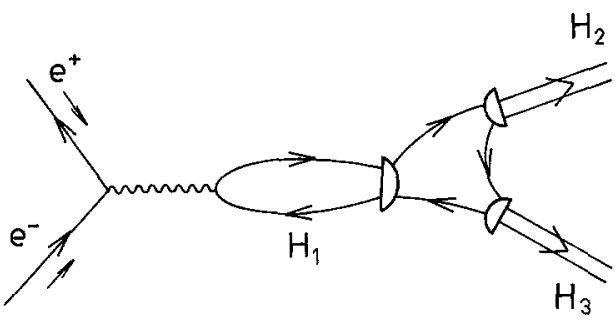

$$
e^{+} e^{-} \rightarrow \mathrm{H}_{2} \mathrm{H}_{3}
$$

Fig. 4. Hadron pair production in $e^{+} e^{-}$collisions. The three-hadron form factor with $H_{1}$ off-shell is needed as an input

mechanism can be meaningfully applied even to a moderate $Q^{2}$-regime (before scaling sets in), provided a reliable dynamics is available for the appropriate 3hadron form factor without proliferation of parameters.

Finally, we illustrate in Fig. 4 that the $3 H$ form factor with $H_{1}$ off-shell is relevant to the process $e^{+} e^{-} \rightarrow H_{1}^{*} \rightarrow H_{2}+H_{3}$. We thus expect in general that the general $3 \mathrm{H}$ form factor that we have obtained in this work is useful in treating semi-inclusive, or exclusive, hadron production in $e^{+} e^{-}$collisions, deep-inelastic scattering, and others.

To summarize, we have given an explicit Lorentz-invariant construction of the $3 H$ form factor in terms of the quark-triangle loop under most general conditions of unequal-mass kinematics for the quarks and hadrons, one in which the only dynamical input is a factorized form $D \otimes \phi$ of the hadron-quark vertex function based on an ansatz of Lorentz-covariant instantaneity of the $q \bar{q}$ interaction within each hadron. The structure is expressed as a simple quadrature, free from internal singularities, in which the convergence is provided by a product of $3 D$ wave functions corresponding to the respective hadrons. It is easily adaptable to a large class of electro-weak interactions merely by freezing out the internal structure of one of the participating vertices. In keeping with the generality of this structure we have refrained from any specific applications in this paper, but one on the Sullivan process for the pion structure function is under way.

Acknowledgement. This work was done during the visit of one of us (A.N.M.) to the National Taiwan University. A.N.M. would like to acknowledge the support from the National Science Council of the Republic of China, as well as the warm hospitality of colleagues at the Department of Physics at National Taiwan University. This work was supported in part by the National Science Council of the Republic of China under grant NSC81-0208-M002-36.

\section{References}

1. For a review of this topic see, e.g., Santhanam, I., et al.: Few-Body Systems 7, 141 (1990)

2. Sullivan, J. D.: Phys. Rev. D5, 1732 (1972)

3. For a review of this process see, e.g., Hwang, W-Y. P.: Chin. J. Phys. (Taipei) 30, 617 (1992)

4. Kadychevsky, V.: Nucl. Phys. B6, 125 (1968)

5. Kalinovsky, Yu. L., et al.: Phys. Lett. B231, 288 (1989); Few-Body Systems 10, 87 (1991)

6. Markov, M. A.: Sov. J. Phys. 3, 452 (1940); Yukawa, H.: Phys. Rev. 77, 219 (1950) 
7. Sazdjian, H.: J. Math. Phys. 28, 2618 (1987); Bijtebier, J.: Nuovo Cim. A100, 91 (1988); Crater, H. W., Van Alstine, P.: Phys. Rev. D37, 1982 (1988)

8. Mitra, A. N., Bhatnagar, S.: Int. J. Mod. Phys. A7, 121 (1992)

9. Mitra, A. N., Santhanam, I.: Few-Body Systems 12, 41 (1992)

10. Particle Data Group: Phys. Rev. D45, Part II (1992)

11. Bohm, M., et al.: Nucl. Phys. B50, 397 (1973); Alibaso, C., Schierholtz, G.: Phys. Rev. D10, 961 (1974); Meyer, R. F.: Nucl. Phys. B71, 226 (1974)

12. Chakrabarty, S., et al.: Prog. Part. Nucl. Phys. 22, 43 (1989), Ch. 6

13. Munczek, H. J., Jain, P.: Phys. Rev. D46, 438 (1992); Aoki, K. I., et al.: Phys. Lett. 266B, 467 (1991)

14. Mitra, A. N., et a1.: Int. J. Mod. Phys. E2, 1 (1993)

15. Goldberger, M., Treiman, S. B.: Phys. Rev. 110, 1178 (1958)

16. Singh, N. N., Mitra, A. N.: Phys. Rev. D38, 1454 (1988)

17. Mcfarlane, A. J.: Rev. Mod. Phys. 34, 41 (1962)

18. Nakanishi, N.: Prog. Theor. Phys. Suppl. 43, 1 (1969)

19. See Ch. 7 of ref. [12]

20. Anjos, J., et al.: Phys. Rev. Lett. 65, 2630 (1990)

21. Some references are: Körner, J., Schuler, G.: Z. Phys. C38, 511 (1988); Bauer, M., Wirbel, M.: Z. Phys. C42, 671 (1989); Isgur, N., Scora, D.: Phys. Rev. D40, 1491 (1989); Gilman, F., Singleton, R.: Phys. Rev. D41, 142 (1990)

22. Gupta, K. K., Mitra, A. N.: Phys. Lett. 267B, 11 (1991)

23. Hwang, W-Y. P., Speth, J., Brown, G. E.: Z. Phys. A339, 383 (1991); Hwang, W-Y. P., Speth, J.: Phys. Rev. D45, 3061 (1992)

Received March 29, 1993; accepted for publication May 13, 1993 